\title{
Introduction to the Special Issue: Integrated Assessment Models and the Social Cost of Water Pollution Workshop
}

\author{
David Keiser, Catherine L. Kling, and Daniel J. Phaneuf
}

We are happy to introduce this special issue of Land Economics focused on water quality. The eight papers that follow were commissioned for presentation at a workshop we hosted titled Integrated Assessment Models and the Social Cost of Water Pollution. ${ }^{1}$ The event took place on April 3-5, 2019, at Cornell University, and the papers were submitted for review shortly thereafter.

The 2019 workshop was the second annual event of an ongoing effort to build capacity for understanding how changes in water quality affect society, with the ultimate goal of providing estimates of the social costs of water pollution that are useful for policy analysis across broad spatial scales. This requires moving beyond case study valuation and cost studies, emphasizing instead multidisciplinary research operating at scale and involving economists, ecologists, hydrologists, and related disciplines. It also requires coordination with state and federal agencies, NGOs, and other stakeholders to provide tools that have both scientific rigor and practical usefulness. Our workshop brought together academic economists, ecologists, hydrologists, and agricultural engineers; agency scientists and policy experts; individuals from private sector institutes; and students to hear talks, participate in discussions, and build foundations for future collaborations.

The reference to integrated assessment models (IAMs) in our workshop title serves to emphasize the scale of our ambition for this research community. Estimating the so-

\footnotetext{
${ }^{1}$ For information on the 2019 workshop and the program, visit https://waterpollution.atkinson.cornell.edu/2019conference/. Funding to support the workshop was provided by the Cornell Atkinson Center for Sustainability and the Center for Agricultural and Rural Development at Iowa State University.
}

cial costs of water pollution involves linking the sources of water pollution with their fate and transport in waterways, their impact on downstream ecosystem services, and changes in economic value or costs among affected populations. This requires standing up IAMs with discipline-specific modules that fit together at common spatial and temporal scales. IAMs for climate change and conventional air pollution provide examples of success for this type of initiative. Research communities have been organized around different models with common objectives, and output from these models has helped define the terms of both policy debates and subsequent research. However, the development of IAMs for water quality has lagged behind their counterparts in climate change and air quality. This stems in part from the challenges specific to water as a medium and the comparative absence of organized research communities. Our goal for this project is to encourage development in both of these areas.

The specific papers are examples of progress to date. They include a mix of benefitsfocused IAMs, cost-focused IAMs, and studies that explore specific components needed for integrated assessment. The applications span locations and spatial scales, such as iconic water bodies and their surroundings (Chesapeake Bay and the Great Lakes), a state-level analysis focused on Michigan, a river basin scale application to the Republican River in Kansas/Nebraska, and individual watersheds in Illinois, Massachusetts, and Minnesota. Collectively the studies illustrate the range of research tasks, challenges, and products that define the agenda of research on the social costs of water pollution.

The papers also illustrate the scale of the endeavor and how much work remains. Our 
goal is to continue organizing annual events that will provide an intellectual home for researchers, policy makers, and other stakehold- ers who are interested in this topic. Please feel free to contact us if you are interested in participating. $^{2}$

${ }^{2}$ More information about the project and past and future workshops can be found at https://waterpollution.atkinson. cornell.edu/. 